\author{
GAME THEORY AND \\ MATHEMATICAL ECONOMICS \\ BANACH CENTER PUBLICATIONS, VOLUME 71 \\ INSTITUTE OF MATHEMATICS \\ POLISH ACADEMY OF SCIENCES \\ WARSZAWA 2006
}

\title{
BILATERAL SEQUENTIAL BARGAINING WITH PERFECT INFORMATION AND DIFFERENT PROTOCOLS
}

\author{
ROBERT GOLAŃSKI \\ Department of Mathematical Economy, Warsaw School of Economics \\ Al. Niepodlegtości 162, 02-554 Warszawa, Poland \\ E-mail: robert.golanski@sgh.waw.pl
}

\begin{abstract}
Most research done in the bargaining literature concentrates on the situations in which players get to be proposers alternately, with the first player being the proposer in the first period, the second player being the proposer in the second period, and so on until the cycle ends and the order of proposers is repeated. However, allowing for only this kind of order is a rather simplifying assumption. This paper looks at the situation in which we allow for much more general kind of protocols. We characterize the unique subgame-perfect equilibrium for two players with different discount factors, give a closed-form solution for the equilibrium payoff and finally analyze the properties of the bargaining power of the players as the function of such elements as their discount factors or their relative position and frequency of being proposers within each bargaining cycle.
\end{abstract}

1. Introduction. Within the last twenty years, many game-theoretical papers analyzed the games of sequential bargaining.

The first and simplest game to be analyzed was one researched by Ståhl (1972). In his approach, two players bargain sequentially over a pie, being proposers alternately. What makes the game relatively simple is that Ståhl's game has finite horizon and hence can be easily solved for subgame perfect equilibrium by backward induction.

Rubinstein (1982) makes the bargaining game more complex: allowing for the infinite horizon makes it impossible to use backward induction as straightforwardly as before. However, Rubinstein considers only the simplest kind of the game, in which each player is alternately a proposer and a responder and hence (because of infinite horizon), the game starting in period 3 is essentially the same game as the original one. Using this fact, Rubinstein is able to solve the game for a perfect equilibrium. It turns out that the game has a unique subgame perfect equilibrium (which is also stationary). With discount

2000 Mathematics Subject Classification: Primary 91A05, 91A10.

The paper is in final form and no version of it will be published elsewhere. 
factors equal to $\alpha$ for the first player, $\beta$ for the second player and the size of the cake equal to one, the outcome of the game is that the first player (the original proposer) will propose an offer in which she keeps $\frac{1-\beta}{1-\alpha \beta}$ for herself and offers the rest to the second player and the offer is accepted.

Alternative proof of the same proposition has been provided by Shaked and Sutton (1984).

As shown by Binmore and Dasgupta (1987), if discount factors are close to 1, the solution of this game is close to Nash bargaining solution.

Since then the extensions of bargaining model consisted mostly of papers focusing on either multilateral bargaining or bargaining with incomplete information. This paper will take a look at bilateral infinite sequential bargaining with perfect information, when a possibility of both discount factors different for each player as well as more complicated protocols determining the order of being a proposer will be allowed.

Until recently all of the papers concentrated on situations in which players were assumed to be proposers sequentially and alternately (at least to some extent, as different papers offer different solutions to the cases when - in multilateral bargaining - some players accept an offer made by the proposer while others reject it). One paper which does not make this kind of assumption about the regularity of the protocol is the paper by Huang (1998). Huang's paper focuses on a procedure for multilateral bargaining, which in particular allows any kind of cyclical protocol (with finite periodicity). The results are easily applicable to bilateral bargaining and indeed, the conclusions that can be drawn from Huang's work for 2-player case are a special case of what we discuss in this paper (namely, when discount factors are equal). Similarly, Rubinstein's paper is a special case of what we analyze, namely the situation when the protocol is the simplest possible (players being proposers alternately).

2. The game. One interesting extension of two-player Rubinstein's game would be looking at the sequential bargaining in which we do not restrict ourselves to situations in which both players are proposers alternately.

The game is set up as typical bargaining problem discussed elsewhere in the literature:

- Players: There are 2 players, labeled $A$ and $B$. In every round of the game one of the players is a proposer and the other is a responder.

- Actions and strategies: There are two kinds of actions: offers, made by a proposer, consisting of a pair of real numbers $(x, y)$ such that $0 \leq x \leq 1,0 \leq y \leq 1$ and $x+y=1^{1}$; and responses, made by a responder, from the set $\{Y, N\}$, where $Y$ denotes accepting the offer and $N$ denotes rejecting it. The strategy will be a contingent plan, describing for each of the players what she should do in every decision node. Notice in particular that we do not restrict our attention to stationary strategies. We allow actions in a given strategy to be conditional on the previous history of the game and on the number of a period in which given action is taken. Even without this restriction, we

\footnotetext{
${ }^{1}$ We therefore assume that the pie has the size 1 . However, this is only a normalizing assumption. We can think of $x$ and $y$ as shares in the pie of any positive size; the results we obtained would then have to be simply changed proportionately to the size of the pie.
} 
will be able to show that the game has a unique subgame perfect equilibrium (which also happens to be stationary).

- Outcomes and payoffs: When an offer $(x, y)$ is made by a proposer and accepted by a responder, player $A$ gets $x$ and player $B$ gets y (equal to $1-x$, notice that the size of the pie does not shrink as the bargaining continues). However, even though in each period the whole pie is divided, players discount the outcomes using, possibly different, discount factors $\alpha$ and $\beta$ for player $A$ and $B$, respectively. Discount factors are nonnegative and strictly below 1 . Players have selfish preferences in the sense that they care only about their own payoffs and not about payoffs of their opponent. Hence, if the agreement is reached for the first time in period $t$, payoffs that players get are equal to $u_{A}=\alpha^{t-1} x$ and $u_{B}=\beta^{t-1} y$ for player $A$ and $B$, respectively.

- Information: The game is of complete and perfect information. Both players have full information about the structure of the game, the history of previous moves, know and remember all past actions.

- Timing: This element is new to the model. Following Huang's paper on multilateral bargaining we set up the game in the following way: assume the game is cyclical with periodicity $p<\infty$ (that is, each cycle consists of $p$ rounds). Let $P=\{1, \ldots, p\}$. The protocol will be the function specifying who is the proposer in every round of the cycle: $\pi: P \rightarrow\{A, B\}$. The regular protocol analyzed by Rubinstein would hence be $\pi(1)=A, \pi(2)=B$ with $p=2$.

We will also represent the protocol by $p$-dimensional vector $\pi^{*}=(\pi(1), \pi(2), \ldots, \pi(p))$. Without loss of generality, we can assume that $A$ moves first (i.e. $\pi(1)=A$ for any $\pi$ ). In the further analysis, we will also assume $\pi$ is onto (each player is a proposer at least once during the game. If it is not true, then because of selfish preferences of the players, in the equilibrium the proposer will get the whole pie in the first round and the game will $\mathrm{end}^{2}$ ).

3. Equilibrium of the game. Before we show the equilibrium of the game, we will solve the following system of equations.

Consider any two consecutive periods, $t$ and $t+1$.

Let $\left(z_{i}, 1-z_{i}\right)$ denote the offer made in period $i \in\{1,2, \ldots, p\}$. Let these offers be such that for any period when $A$ is a proposer we have $z_{t}=1-\beta\left(1-z_{t+1}\right)$ or equivalently that $z_{t}-\beta z_{t+1}=1-\beta$ and for any period when $B$ is a proposer we have $z_{t}=\alpha z_{t+1}$ or equivalently $z_{t}-\alpha z_{t+1}=0$. In order to find a solution in a general case, we will have

\footnotetext{
${ }^{2}$ It is easy to see that this is actually the case. Notice that any subgame following the rejection by a responder is (because of an infinite and periodic nature of the game) identical to the subgame preceding the rejected offer. Hence, it must be that the supremum $S$ of what player $B$ will get in any subgame in any equilibrium must be the same in both these subgames (as they are identical). On the other hand, player $A$ will never offer player $B$ more than $\beta S$ since any higher offer would certainly be accepted and there would always exist a lower offer, making $A$ better off. Hence, we get that $S$ must be such that $S \leq \beta S$. But since by assumption $\beta<1$ and only nonnegative offers are allowed, it must be that $S=0$ and hence $B$ must get 0 -share in any subgame perfect equilibrium in the game in which $A$ is a proposer in every period
} 
to construct the system of $p$ equations (one for each period in the cycle) and solve it for $z_{i}$ 's. Let $z$ denote the column vector of $z_{i}$ 's.

We can write the system of equations that we want to solve in the following matrix form $M z=N$, where (as is evident from the way these equations are described above) $M$ is a $p \times p$ matrix with the following properties:

- each row corresponding to the period when $A$ is a proposer has 1 on the main diagonal, $-\beta$ to the right of it (or in the case of the last period, it has 1 in the last column and $-\beta$ in the first column) and 0's elsewhere;

- each row corresponding to the period when $B$ is a proposer has 1 on the main diagonal, $-\alpha$ to the right of it and 0's elsewhere.

and $N$ is a $p \times 1$ column vector with the following property:

- it has $1-\beta$ on positions corresponding to $A$ being the proposer;

- it has 0's on positions corresponding to $B$ being the proposer.

We can now find $z_{i}$ using Cramer's rule. In order to find $z_{1}$, we must calculate two things:

- determinant of $M$;

- determinant of $M$ with its first column replaced by $N$ (denoted from now on by $M \backslash N$ ).

3.1. Determinant of $M$. We will expand the matrix $M$ using its first column and Laplace rule of expansion of cofactors. Notice that $M$ has non-zero expressions in the first column only in the first and the last row. It's easy to see that matrices formed by deleting the first column and either first or last row are triangular and hence their determinants are equal to the product of the expressions on their diagonal (and all other cofactors corresponding to other rows are equal to zero).

Let $P_{A}=\{i$ : player $A$ is a proposer and $i \in P\}$. Let $P_{B}=P \backslash P_{A}$. Hence it must be that $|M|=1-\alpha^{\left|P_{B}\right|} \beta^{\left|P_{A}\right|}$ where one corresponds to cofactor obtained from deleting the first row and the first column of $M$ (which leaves only 1's on the main diagonal) and $-\alpha^{\left|P_{B}\right|} \beta^{\left|P_{A}\right|}$ corresponds to the cofactor obtained from deleting the last row and the first column (notice that if $p$ is even, the product of the expressions on the main diagonal of the reduced matrix will be the product of odd number of negative terms, so it will be negative itself. The cofactor is then achieved by multiplying this product by one more negative term (corresponding to either $-\alpha$ or $-\beta$ being $M_{p, 1}$ element of the matrix $M$ ) and by $(-1)^{p+1}$, which with $p$ even is also negative and so the whole expression is negative. On the other hand, if $p$ is odd, we still will get the negative cofactor because even though the determinant of reduced matrix is positive it is now multiplied by negative term of $-\alpha$ or $-\beta$ and positive term of $\left.(-1)^{p+1}\right)$.

3.2. Determinant of $M \backslash N$. Also in this case we will expand the matrix by the first column. Notice the following:

- the first column $(=N)$ will now contain only 0's or $1-\beta$,

- the matrix resulting from deleting first column and $i$-th row need not be triangular anymore. However, it has a nice property of having $-\alpha$ 's and $\beta$ 's above the deleted row and 1 below it on the main diagonal. 
Notice that unlike in the situation where we were calculating the determinant of M, now the matrices formed by deleting first column and $t$-th row need not be triangular and hence finding the determinant of such a matrix will be more complicated. As it turns out, however, $M \backslash N$ still has such a structure that its determinant can be found simply by taking the product of the expressions on the main diagonal, as if it was a triangular matrix. Suppose $A$ is a proposer in period $t$. Then matrix $M \backslash N$ will have $1-\beta$ as its ( $t$-th row, first column) element. If we delete the first column and the $t$-th row (which will have to be done in order to find the necessary cofactor), the resulting matrix will look as follows:

- in the rows corresponding to periods preceding $t$, there will be $-\alpha$ 's and $-\beta$ 's on the main diagonal and 1's to the left of it;

- in the rows corresponding to the periods following $t$, there will be 1 's on the main diagonal and $-\alpha$ 's and $-\beta$ 's to the right of it.

Therefore, the determinant of the $M \backslash N$ matrix will be given by the sum of the expressions, each of which will be a product of $(1-\beta)$ and the product of as many $\alpha$ 's as many times $B$ is the proposer in the periods preceding a given turn and as many $\beta$ 's as many times $A$ is the proposer in the preceding periods.

Combining these two results we get that

$$
z_{1}=\frac{(1-\beta) \sum_{i \in P_{A}} \alpha^{\left|P_{B} \cap R_{i}\right|} \beta^{\left|P_{A} \cap R_{i}\right|}}{1-\alpha^{\left|P_{B}\right| \beta^{\left|P_{A}\right|}}}
$$

where $R_{i}=\{0,1, \ldots, i-1\}$.

As we can see, the sum in the numerator is the sum of $\left|P_{A}\right|$ terms, each corresponding to one period in the cycle in which $A$ is a proposer and each being a product of discount factors equal to player $A$ 's discount factor to the power of how many times player $B$ was a proposer in the previous periods times player $B$ 's discount factor to the power of how many times player $A$ was a proposer in the previous periods. For example, with the protocol $(A, B, A, A, B)$, the sum would be equal to $\alpha^{0} \beta^{0}+\alpha^{1} \beta^{1}+\alpha^{1} \beta^{2}$ or equivalently $1+\alpha \beta+\alpha \beta^{2}$.

One thing worth noticing is the asymmetry in the way in which players' discount factors are paired with the numbers of their being proposers during the cycle, i.e. the fact that each player's discount factor, in expressions both in the numerator and in the denominator, is raised to the power which is a function of the other player's position as a proposer.

It would be also worth noticing that we can express (2) in another way. Notice in particular the exponent at $\beta$ in the numerator sum. As it depends on the number of periods player $A$ is the proposer and since the sum is taken over all periods when $A$ is the proposer, the sum in the numerator will have the property that in each expression $\beta$ will be raised to the power 1 higher than in the previous expression. Also, the sum of exponents at $\alpha$ and $\beta$ at each term of the sum must be equal to the number of a corresponding period in the cycle diminished by one (since the sum of exponents corresponds to the number of times either player was a proposer in the preceding periods, which is naturally the same as the number of preceding periods itself). Hence, if we denote the periods in 
which player $A$ is the proposer during the cycle by $1=t_{1}<t_{2}<\ldots<t_{k} \leq p$, the expression given by (2) is the same as

$$
z_{1}=\frac{(1-\beta) \sum_{i=1}^{k} \alpha^{t_{i}-i} \beta^{i-1}}{1-\alpha^{p-k} \beta^{k}}
$$

We can now formally characterize the equilibrium of the game.

THEOREM. The only subgame perfect equilibrium of the game described in Section 2 is one in which:

- in period $t$, a proposer offers a split $\left(z_{t \bmod p}, 1-z_{t \bmod p}\right)$ with $z_{0}=z_{p}$.

- in period $t$, player $A$ accepts if offered at least $z_{t \bmod p}$, player $B$ accepts if offered at least $1-z_{t \bmod p}$.

Proof. The idea of the proof follows Shaked and Sutton (1984) and Muthoo (1999) for a regular protocol $(A, B)$ and is as follows:

Let $S_{i}^{A}, S_{i}^{B}$ denote suprema of payoffs that players $A$ and $B$, respectively, can receive in any subgame perfect equilibrium starting in period i, and similarly, let $I_{i}^{A}, I_{i}^{B}$ denote infima of payoffs that players $A$ and $B$, respectively, can receive in any subgame perfect equilibrium starting in period $i$.

Consider any two consecutive periods $i$ and $i+1$ such that player $A$ is the proposer in period $i$. Player $A$ knows that any offer below discounted value of player $B$ 's next round infimum (that is any offer such that player $B$ is offered less than $\beta I_{i+1}^{B}$ ) must be necessarily rejected in any equilibrium (since otherwise $B$ would not be best-responding), so we must have that $S_{i}^{A} \leq 1-\beta I_{i+1}^{B}$. Suppose though that $S_{i}^{A}<1-\beta I_{i+1}^{B}$. Then there must exist subgame perfect equilibrium payoff to player $B$ equal to $u$ such that $S_{i}^{A}<1-\beta u$. But by definition of a supremum, it follows that there are no subgame perfect equilibria in which player $A$ gets the share of $1-\beta u$. But $u$ can be supported as the equilibrium outcome by the pair of strategies in which the split offered in the previous round is $x=1-\beta u$ and responders will only accept offers at least as good. But then player $A$ gets $1-\beta u$ in such an equilibrium, which contradicts the fact that $S_{i}^{A}<1-\beta I_{i+1}^{B}$. So it follows that we must have $S_{i}^{A}=1-\beta I_{i+1}^{B}$.

Similarly we can argue that $I_{i}^{A}=1-\beta S_{i+1}^{B}$, and for every period $i$ in which player $B$ is the proposer we similarly get $I_{i}^{B}=1-\alpha S_{i+1}^{A}$ and $S_{i}^{B}=1-\alpha I_{i+1}^{A}$.

Now notice that the game starting in period $p+1$ is exactly the same as the game starting in period 1, so they must have the same equilibria, and so suprema and infima of payoffs that players get in these two games must be identical, so $S_{1}^{A}=S_{p+1}^{A}$ and $I_{1}^{A}=I_{p+1}^{A}$.

Notice also that in any equilibrium the whole good must be divided because players care only about their own share. If there was an equilibrium in which not all good was divided, then the proposer might take the remaining share and improve her utility, so the split less than full cannot happen in equilibrium. It must therefore be that for any $i$, $S_{i}^{A}=1-I_{i}^{B}$ and $I_{i}^{A}=1-S_{i}^{B}$.

We can therefore express the above conditions using as unknowns suprema and infima of payoffs of player $A$ only-for any period $i$ in which player $A$ is the proposer we get $S_{i}^{A}=1-\beta I_{i+1}^{B}=1-\beta\left(1-S_{i+1}^{A}\right)$ and therefore $S_{i}^{A}-\beta S_{i+1}^{A}=1-\beta$ and similarly for any 
period $i$ in which player $B$ is the proposer we get $I_{i}^{B}=1-S_{i}^{A}=1-\alpha S_{i+1}^{A}$ and therefore $S_{i}^{A}-\alpha S_{i+1}^{A}=0$. But if so, the solution to vector $\left\{S_{i}\right\}_{i=1}^{p}$ is given by expressions found in the previous section. In particular,

$$
S_{1}^{A}=\frac{(1-\beta) \sum_{i \in P_{A}} \alpha^{\left|P_{B} \cap R_{i}\right|} \beta^{\left|P_{A} \cap R_{i}\right|}}{1-\alpha^{\left|P_{B}\right| \beta^{\left|P_{A}\right|}} .}
$$

Similarly, using the expressions with infima of payoffs of player $A$ only we can solve in the same way for all $I_{i}^{A}$, and in particular we can find that

$$
I_{1}^{A}=\frac{(1-\beta) \sum_{i \in P_{A}} \alpha^{\left|P_{B} \cap R_{i}\right|} \beta^{\left|P_{A} \cap R_{i}\right|}}{1-\alpha^{\left|P_{B}\right| \beta^{\left|P_{A}\right|}} .}
$$

But if so, we get that $S_{1}^{A}=I_{1}^{A}$, so it must be that in any subgame perfect equilibrium the equilibrium payoff will be equal to

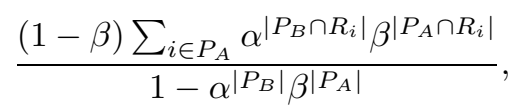

and in a similar manner we can argue that in any equilibrium the split offered in period $t$ must be equal to $z_{t \bmod p}, 1-z_{t \bmod p}$ (with $z_{0}=z_{p}$ ), where $z_{i}$ 's are solutions to the equation system solved in the previous section.

Notice further that the same argument can be used for calculating the equilibrium payoff in any subgame starting at any given period $t$ (the unique equilibrium offer must be $\left(z_{t \bmod p}, 1-z_{t \bmod p}\right)\left(\right.$ with $\left.\left.z_{0}=z_{p}\right)\right)$. It's easy to see that the described part of strategies hence supports vector $z$ as equilibrium partition for a given period. Also, there is no other pair of strategies that would support it as the equilibrium: by the argument about suprema and infima, no other offer can be made on the equilibrium path and similarly, since players are best responding, they will always accept offers higher than the described equilibrium offers and reject offers lower than those.

There is one thing about the equilibrium payoff that is worth mentioning. Even though we made the assumption that player $A$ is the first proposer in the game and made all the calculations based on this fact, it turns out that the expression for the equilibrium payoff for player $B$ can also be described in the same manner: it is the product of $1-\alpha(=$ the other player's discount factor) and the sum which assigns to each period in which $B$ is a proposer an expression which is the product of $\alpha$ 's raised to the number of periods in which $B$ was a proposer before and $\beta$ 's raised to the number of periods in which $A$ was a proposer before, all divided by $1-\alpha^{\left|P_{B}\right|} \beta^{\left|P_{A}\right|}$. In order to prove this claim we will have to show that the sum of these two expressions is equal to 1 . Hence we have

$$
\begin{aligned}
& \frac{(1-\beta) \sum_{i \in P_{A}} \alpha^{\left|P_{B} \cap R_{i}\right|} \beta^{\left|P_{A} \cap R_{i}\right|}}{1-\alpha^{\left|P_{B}\right|} \beta^{\left|P_{A}\right|}}+\frac{(1-\alpha) \sum_{i \in P_{B}} \alpha^{\left|P_{B} \cap R_{i}\right|} \beta^{\left|P_{A} \cap R_{i}\right|}}{1-\alpha^{\left|P_{B}\right|} \beta^{\left|P_{A}\right|}} \\
= & \frac{\sum_{i \in P_{A}} \alpha^{\left|P_{B} \cap R_{i}\right|} \beta^{\left|P_{A} \cap R_{i}\right|}-\beta \sum_{i \in P_{A}} \alpha^{\left|P_{B} \cap R_{i}\right|} \beta^{\left|P_{A} \cap R_{i}\right|}}{1-\alpha^{\left|P_{B}\right|} \beta^{\left|P_{A}\right|}} \\
& +\frac{\sum_{i \in P_{B}} \alpha^{\left|P_{B} \cap R_{i}\right|} \beta^{\left|P_{A} \cap R_{i}\right|}-\alpha \sum_{i \in P_{B}} \alpha^{\left|P_{B} \cap R_{i}\right|} \beta^{\left|P_{A} \cap R_{i}\right|}}{1-\alpha^{\left|P_{B}\right|} \beta^{\left|P_{A}\right|}} \\
= & \frac{\sum_{i \in P} \alpha^{\left|P_{B} \cap R_{i}\right|} \beta^{\left|P_{A} \cap R_{i}\right|}-\beta \sum_{i \in P_{A}} \alpha^{\left|P_{B} \cap R_{i}\right|} \beta^{\left|P_{A} \cap R_{i}\right|}-\alpha \sum_{i \in P_{B}} \alpha^{\left|P_{B} \cap R_{i}\right|} \beta^{\left|P_{A} \cap R_{i}\right|}}{1-\alpha^{\left|P_{B}\right|} \beta^{\left|P_{A}\right|}} .
\end{aligned}
$$


But now notice the following: the first expression in the numerator has one term for each period of the cycle. The first of these terms will be equal to 1 , which is equal to $\alpha^{0} \beta^{0}$ (both powers are zero since no one was to be a proposer prior to period 1 ). On the other hand, all other expressions of this sum cancel with one of the expressions of two other sums, namely with the expression corresponding to the preceding period: the term in the first sum is equal to the product of $\alpha$ 's to the power of how many times $B$ was the proposer before and $\beta$ 's to the power of how many times $A$ was a proposer before, the term in one of two other sums corresponding to the preceding period will be equal (after being multiplied by $\alpha$ or $\beta$ standing before these sums) to exactly the same amount. Hence, because only players $A$ and $B$ get to be proposers in any period and one and only one of them must be a proposer in each period, the only two terms that will not cancel out in the numerator will be 1 corresponding to the first period in the first sum and the expression in one of two latter sums, corresponding to the last period (since there is no period in the cycle following the last period and hence, there is no term in the first sum that this last term could get canceled with). This last expression that will not get canceled out, will be equal to the product of $\alpha$ to the power of how many times $B$ was the proposer in all but last periods times $\beta$ to the power of how many times $A$ was the proposer in all but last periods, all multiplied by negative $\beta$ if $A$ is the last proposer or negative $\alpha$ if $B$ is the last proposer.

But notice that if so, this expression is equal exactly to $\alpha^{\left|P_{B}\right|} \beta^{\left|P_{A}\right|}$ and hence the whole sum given by expression (4) is equal to 1 . Therefore, the equilibrium payoff of both players can be characterized in exactly the same way and easily determined from the protocol.

4. Properties of the equilibrium outcome. Properties of the equilibrium outcome are quite intuitive:

4.1. $z_{1}$ increases with $\alpha$. One of the properties that we would expect of the bargaining equilibrium payoff would be that as the player becomes more and more patient (i.e. the discount factor she uses to evaluate the value of the payoff she gets), her bargaining power should increase. In particular that would mean that as $\alpha$ increases, $z_{1}$ should also increase. Let

$$
W(\alpha, \beta, \pi)=\sum_{i \in P_{A}} \alpha^{\left|P_{B} \cap R_{i}\right|} \beta^{\left|P_{A} \cap R_{i}\right|}=\sum_{i=1}^{k} \alpha^{t_{i}-i} \beta^{i-1}
$$

Then we have

$$
\begin{aligned}
\frac{\partial z_{1}}{\partial \alpha} & =(1-\beta) \frac{\left(\frac{\partial W}{\partial \alpha}\right)\left(1-\alpha^{\left|P_{B}\right|} \beta^{\left|P_{A}\right|}\right)+W\left|P_{B}\right| \alpha^{\left|P_{B}\right|-1} \beta^{\left|P_{A}\right|}}{\left(1-\alpha^{\left|P_{B}\right|} \beta^{\left|P_{A}\right|}\right)^{2}} \\
& =(1-\beta) \frac{\frac{\partial W}{\partial \alpha}-\frac{\partial W}{\partial \alpha} \alpha^{\left|P_{B}\right|} \beta^{\left|P_{A}\right|}+W\left|P_{B}\right| \alpha^{\left|P_{B}\right|-1} \beta^{\left|P_{A}\right|}}{\left(1-\alpha^{\left|P_{B}\right|} \beta^{\left|P_{A}\right|}\right)^{2}} \\
& =(1-\beta) \frac{\frac{\partial W}{\partial \alpha}+\alpha^{\left|P_{B}\right|-1} \beta^{\left|P_{A}\right|}\left(W\left|P_{B}\right|-\alpha \frac{\partial W}{\partial \alpha}\right)}{\left(1-\alpha^{\left|P_{B}\right|} \beta^{\left|P_{A}\right|}\right)^{2}} .
\end{aligned}
$$

But now notice the following:

- Since $\beta$ is bounded by 1 from above, $1-\beta$ is positive; 
- $\left(1-\alpha^{\left|P_{B}\right|} \beta^{\left|P_{A}\right|}\right)^{2}$, being a square of non-zero expression (which is assured by the fact that we require $\alpha$ and $\beta$ to be strictly below 1 ), must also be positive;

- $\frac{\partial W}{\partial \alpha}=\sum_{i=1}^{k}\left(t_{i}-i\right) \alpha^{t_{i}-i-1} \beta^{i-1}$ which is nonnegative $\left(t_{i} \geq i\right.$ since $A$ can be proposer for the $i$-th time not sooner than in the $i$-th period);

- Finally, notice that

$$
W\left|P_{B}\right|=\sum_{i=1}^{k}(p-k) \alpha^{t_{i}-i} \beta^{i-1}
$$

and

$$
\alpha \frac{\partial W}{\partial \alpha}=\sum_{i=1}^{k}\left(t_{i}-i\right) \alpha^{t_{i}-i} \beta^{i-1}
$$

that is, both expressions are sums of products of $\alpha$ 's and $\beta$ 's raised to the same power and differ only by coefficients standing at each of its terms. Notice however by definition, $t_{i}-i$ cannot be higher than $p-k$ for any $i$ and hence, all coefficients in (8) are (weakly) lower than coefficients in (7). Therefore, also the last expression that we had to look at must be nonnegative.

But since (6) is the sum and product of nonnegative expressions, it must itself be nonnegative (and in fact, with $\beta<1$, it is always strictly positive unless $k=1$ and $\alpha$ or $\beta$ are equal to zero). Therefore, the bargaining power of the player, expressed by the pie share that she gets in the equilibrium, increases as the player becomes more patient.

4.2. $z_{1}$ decreases with $\beta$. Because of the $(1-\beta)$ factor in the expression for $z_{1}$, the actual calculation of the $\frac{\partial z_{1}}{\partial \beta}$ derivative and checking whether this derivative is negative for all admissible values of $\alpha$ and $\beta$ and all possible protocols is not an easy task. There is however an easier way to see that the above claim is actually true and that the bargaining power of a player decreases as her opponent becomes more patient.

Notice the following: as we have shown in section 3, the expressions for the equilibrium outcomes of both players are symmetrical and calculated in the same manner. Hence, since the argument showing that $z_{1}$ would increase with $\alpha$ did not rely in any part on the fact that $A$ is the original proposer, it must also be true that for the original responder (i.e. for player $B$ ), his equilibrium payoff will increase with his discount factor. Therefore, by the same argument as before, we may argue that $1-z_{1}$ will increase with $\beta$, which directly implies that $z_{1}$ itself will decrease with $\beta$.

4.3. Informally, the sooner one is the proposer, the higher her power (if we switch a period when $A$ was a proposer with a period when $B$ is a proposer so that now $A$ is a proposer in an earlier period, $z_{1}$ will increase).

More formally, let $\pi$ be any protocol. Let $\pi_{1}$ be a protocol formed from $\pi$ in the following way:

- for some $i$ and $j$ in $P(i<j), \pi(i)=\pi_{1}(j)=B, \pi(j)=\pi_{1}(i)=A$;

- for all $k \neq i, j, \pi(k)=\pi_{1}(k)$.

Then $z_{1}$ is (weakly) higher under $\pi_{1}$ than under $\pi$.

In order to verify that this is true, we will have to compare the expressions for $z_{1}$ under both protocols. Notice however that in the expression for $z_{1}$ (given by (2) or (3)), 
$1-\beta$ is the same under both protocols and the denominator is also the same under both protocols (which is because under both protocols $A$ (or $B$ ) is a proposer the same number of times and what matters for the expression in the denominator is only the number of times each player gets to be a proposer and not their relative order within the cycle).

Therefore, since these two expressions are equal (and positive) under both protocols, in order to establish that $z_{1}$ is higher under $\pi_{1}$ than under $\pi$, it is enough to show that $W\left(\alpha, \beta, \pi_{1}\right)>W(\alpha, \beta, \pi)$ or equivalently that $W\left(\alpha, \beta, \pi_{1}\right)-W(\alpha, \beta, \pi)>0$.

Notice now, that all terms in $\mathrm{W}$ are the products of $\alpha$ and $\beta$ raised to the appropriate power corresponding to the number (but not order) of one of the player's being the proposer in all periods preceding the period for which a given product is taken. Therefore, in both $W\left(\alpha, \beta, \pi_{1}\right)$ and $W(\alpha, \beta, \pi)$ all expressions corresponding to periods prior to $i$ will be the same (since there is no change prior to $i$ ), and also all expressions corresponding to periods following $j$ will be the same (since the only thing that changes prior to these periods is the order in which $A$ and $B$ are proposers, but not the total number of times each of them will make the offer). Hence, it is enough to consider changes between period $i$ and $j$.

Let $i<\tau_{1}<\ldots<\tau_{m}=j$ denote periods between $i$ and $j$ in which player $A$ is a proposer under a protocol $\pi$. Then we must have that under $\pi_{1}$ player $A$ will be the proposer in periods $\tau_{0}=i, \tau_{1}, \ldots, \tau_{m-1}$. Let $\varphi=\alpha^{\mu} \beta^{\nu}$ where $\mu$ is the number of periods player $B$ was a proposer prior to $i$ and $\nu$ is the number of periods player $A$ was a proposer prior to $i$. Then the difference whose sign we have to check will be given by

$$
\begin{aligned}
\alpha^{\mu} \beta^{\nu}\left(\sum_{n=0}^{m-1} \alpha^{\tau_{n}-\tau_{0}-n} \beta^{n}\right)-\alpha^{\mu} \beta^{\nu} & \left(\sum_{n=1}^{m} \alpha^{\tau_{n}-\tau_{0}-n+1} \beta^{n-1}\right) \\
= & \alpha^{\mu} \beta^{\nu} \sum_{n=1}^{m}\left(\alpha^{\tau_{n-1}-\tau_{0}-n+1} \beta^{n-1}-\alpha^{\tau_{n}-\tau_{0}-n+1} \beta^{n-1}\right) \\
& =\alpha^{\mu} \beta^{\nu} \sum_{n=1}^{m} \alpha^{\tau_{n-1}-\tau_{0}-n+1} \beta^{n-1}\left(1-\alpha^{\tau_{n}-\tau_{n-1}}\right) .
\end{aligned}
$$

But now, since by construction $\tau_{n}-\tau_{n-1}>0$, it must be that $1-\alpha^{\tau_{n}-\tau_{n-1}}>0$ as well and hence the whole difference must be also positive. Therefore, the player cannot lose by this kind of swapping positions in a way that she gets to be a proposer in earlier periods.

Using the same argument in the opposite direction, we can argue that the player cannot benefit by the situation in which the swap in the order moves her towards the end of the cycle. Also, by repeating the same argument, if we make a number of swaps, in each of which the same player will be moved closer to the beginning of the cycle, this player will benefit of these changes. As a corollary to this we have the following result: if the game cycle has a length of $p$ and player $A$ is a proposer in $k$ of $p$ periods in each cycle, she will get the highest equilibrium payoff under the protocol in which $P_{A}=\{1,2, \ldots, k\}$ and $P_{B}=\{k+1, k+2, \ldots, p\}$.

Notice also that this result does not imply - unlike in the original Rubinstein modelthat the bargaining power of the first proposer is higher than that of the first responder. It may be beneficial to be the responder in the first period if one is a proposer in sufficiently many following periods. 
4.4. Informally, the more often one is a proposer, the higher her bargaining power (replacing $B$ with $A$ in the protocol for some periods while keeping protocol intact for all periods when $A$ was an original proposer, increases $z_{1}$ ).

One property that we would also expect of the bargaining power is that it should increase if the player gets to be a proposer more often. Of course, because of discount factors this will not hold unconditionally. Suppose for example that $\alpha=0$. Then player $A$ will only care about how much she gets in the first period of the game, since if the game does not reach the agreement then, player $A$ will get zero-utility no matter when the game will end and what split of the pie will be adopted. But if so, player with zero discount factor can get positive equilibrium payoff only if she is the original proposer. Otherwise, no matter what the protocol is, there is no reason for the original proposer to make any positive offer, since there is always lower offer that is still going to be accepted by the player with zero-discount factor and since there is no lowest positive number, the only offer that could be sustained as the equilibrium would be $z_{1}=1$. In fact, using (2), it is easy to see that if $\alpha=0$, then no matter how big $p$ is and even if $A$ is the proposer only in the first period of the cycle, we will get that

$$
z_{1}=1-\beta
$$

which is strictly positive as long as $\beta<1$.

On the other hand, if $\beta=0$, then even if player $B$ is the proposer in each but the first period of the cycle, as is apparent from (3) and (4), what he gets in the equilibrium is equal to

$$
1-z_{1}=0
$$

Hence, player with zero discount factor is better off by being the proposer only once but at the very beginning of the game then being the proposer for all other periods of the cycle if she is the responder in the first period.

What we postulate is hence not that the player benefits by the mere fact that she gets to be a proposer more often but rather that the player cannot lose if for the given protocol she will get more periods when she is the proposer while still being a proposer in all periods in which she was the proposer under the original protocol.

Let $\pi: P \rightarrow\{A, B\}$ be some protocol and let $\pi_{1}$ be the protocol identical to $\pi$ except for one period in which we replace $B$ by $A$. More formally, we have that there exists $j \in P$ such that $\pi(j)=B, \pi_{1}(j)=A$ and $\pi_{1}(i)=\pi(i)$ for all $i \neq j, i \in P$. Then the equilibrium payoff of player $A$ is higher under protocol $\pi_{1}$ than under protocol $\pi$.

Although this property is both desirable and quite intuitive, it is not at all obvious from the form of the solution that we got in (2). In particular, notice that if we replace $B$ by $A$ for one of the periods of the cycle, then the following will happen:

- the terms in the sum given by expression (5) for the periods earlier than $j$ will not change;

- there will appear an additional positive (and strictly positive, if both $\alpha$ and $\beta$ are strictly positive) term in the above sum, corresponding to the fact that $A$ gets to be a proposer one more time under $\pi_{1}$ than under $\pi$; 
- the terms corresponding to the periods following $j$ under $\pi_{1}$ will be equal to the terms under $\pi$ multiplied by $\frac{\beta}{\alpha}$ (which reflects the fact that for any such period, $B$ is now a proposer one period fewer in the preceding periods and $A$ is a proposer one period more). In particular, that would mean that if $\alpha>\beta$, each of these terms is actually higher under $\pi$ than under $\pi_{1}$ (or, in other words, even though player $A$ benefits from the facts that she is the proposer in one additional period, if $\alpha>\beta$ it is not clear that her total equilibrium payoff will actually increase);

- moreover, since under the new protocol player $A$ is actually the proposer one time more often, the denominator in (2) will also change to become $1-\alpha^{\left|P_{B}\right|-1} \beta^{\left|P_{A}\right|+1}$ under $\pi_{1}$ as compared to $1-\alpha^{\left|P_{B}\right|} \beta^{\left|P_{A}\right|}$ under $\pi^{3}$. If $\alpha>\beta$, this denominator will increase, causing a decrease in the equilibrium payoff under $\pi_{1}$ protocol).

It is clear from the above reasoning that if $\alpha<\beta, z_{1}$ will increase if we make $A$ a proposer in one more period. What we still have to show is that even if $\alpha>\beta$ the increase in $z_{1}$ resulting from the fact that one more term (corresponding to one more period when $A$ is the proposer) is added, compensates for the decrease resulting from the two other effects described above.

However, showing it in the general case is rather complicated. Rather than trying to prove the result for the general situation, we will just show that a player benefits even if she becomes the proposer under $\pi_{1}$ in the last period of the cycle in which her opponent was a proposer under $\pi$ and then we will argue that this implies the result stated in subsection 4.4.

Because of the identical way the equilibrium payoffs are determined for both players, as shown in (4), without loss of generality we can focus on one of the players, say player $A$. We want to show that adding one more period in which player $A$ will be a proposer must (weakly) increase $z_{1}$.

Notice that two things may happen: either $A$ or $B$ is the proposer in the last period of the cycle. Suppose player $B$ is the proposer in the last period of the cycle and $\pi_{1}$ differs from $\pi$ only at that last period. Since only the last period is affected, only two things will change in the expression for $z_{1}$ : one more term will be added to the numerator (and all other terms in the sum will remain the same as before since there is no change in the periods prior to periods to which they correspond) and the denominator will change from $1-\alpha^{\left|P_{B}\right|} \beta^{\left|P_{A}\right|}$ to $1-\alpha^{\left|P_{B}\right|-1} \beta^{\left|P_{A}\right|+1}$.

Let $\varphi$ be the sum of all terms in W corresponding to the periods in which player $A$ was the proposer under $\pi$ (and hence is the same under both protocols). Then the difference whose sign we have to check is equal to

$$
\frac{(1-\beta)\left(\varphi+\alpha^{\left|P_{B}\right|-1} \beta^{\left|P_{A}\right|}\right)}{1-\alpha^{\left|P_{B}\right|-1} \beta^{\left|P_{A}\right|+1}}-\frac{(1-\beta) \varphi}{1-\alpha^{\left|P_{B}\right|} \beta^{\left|P_{A}\right|}} .
$$

We need to show that this difference is nonnegative. Notice that this difference is equivalent to

$$
\frac{(1-\beta)\left(\varphi+\alpha^{\left|P_{B}\right|-1} \beta^{\left|P_{A}\right|}\right)\left(1-\alpha^{\left|P_{B}\right|} \beta^{\left|P_{A}\right|}\right)-(1-\beta) \varphi\left(1-\alpha^{\left|P_{B}\right|-1} \beta^{\left|P_{A}\right|+1}\right)}{\left(1-\alpha^{\left|P_{B}\right|-1} \beta^{\left|P_{A}\right|+1}\right)\left(1-\alpha^{\left|P_{B}\right|} \beta^{\left|P_{A}\right|}\right)} .
$$

\footnotetext{
${ }^{3}\left|P_{B}\right|$ and $\left|P_{A}\right|$ in these two expressions stand for the cardinality of $P_{B}$ and $P_{A}$ under $\pi$.
} 
And since both $1-\beta$ and the denominator are positive, it is enough to check the sign of

$$
\begin{aligned}
\left(\varphi+\alpha^{\left|P_{B}\right|-1} \beta^{\left|P_{A}\right|}\right)\left(1-\alpha^{\left|P_{B}\right|} \beta^{\left|P_{A}\right|}\right)-\varphi\left(1-\alpha^{\left|P_{B}\right|-1} \beta^{\left|P_{A}\right|+1}\right) & \\
& =-\alpha^{\left|P_{B}\right|} \beta^{\left|P_{A}\right|} \varphi+\alpha^{\left|P_{B}\right|-1} \beta^{\left|P_{A}\right|}-\alpha^{2\left|P_{B}\right|-1} \beta^{2\left|P_{A}\right|}+\alpha^{\left|P_{B}\right|-1} \beta^{\left|P_{A}\right|+1} \varphi \\
& =\alpha^{\left|P_{B}\right|-1} \beta^{\left|P_{A}\right|}\left(-\alpha \varphi+1-\alpha^{\left|P_{B}\right|} \beta^{\left|P_{A}\right|}+\beta \varphi\right)
\end{aligned}
$$

and again, since $\alpha^{\left|P_{B}\right|-1} \beta^{\left|P_{A}\right|}$ is nonnegative, it is enough to check the sign of the other expression, $-\alpha \varphi+1-\alpha^{\left|P_{B}\right|} \beta^{\left|P_{A}\right|}+\beta \varphi$.

Let $t_{1}, \ldots, t_{k}$ be the periods in which player $A$ is the proposer under $\pi$. Then by definition, $\varphi$ will be equal to $\sum_{i=1}^{k} \alpha^{t_{i}-i} \beta^{i-1}$. But if so, we get that

$$
\begin{aligned}
(\alpha-\beta) \varphi+\alpha^{\left|P_{B}\right|} \beta^{\left|P_{A}\right|}=(\alpha-\beta) \sum_{i=1}^{k} \alpha^{t_{i}-i} \beta^{i-1}+\alpha^{p-k} \beta^{k} & \\
& =\sum_{i=1}^{k} \alpha^{t_{i}-i+1} \beta^{i-1}-\sum_{i=1}^{k} \alpha^{t_{i}-i} \beta^{i}+\alpha^{p-k} \beta^{k}
\end{aligned}
$$

and by grouping the expressions with the same exponents at $\beta$ 's we get that this is equal to

$$
\alpha^{t_{1}}+\sum_{i=1}^{k-1}\left(\alpha^{t_{i+1}-i}-\alpha^{t_{i}-i}\right) \beta^{i}+\left(\alpha^{p-k}-\alpha^{t_{k}-k}\right) \beta^{k} .
$$

But now notice that:

- Since $\alpha$ is less than 1 and $t_{1}$ is at least equal to 1 , it must be that $\alpha^{t_{1}}<1$;

- Since by construction $t_{i+1}>t_{i}$, it must be that $\alpha^{t_{i+1}-i}<\alpha^{t_{i}-i}$ for any $i$ and similarly since $p>t_{k}$, we must have $\alpha^{p-k}<\alpha^{t_{k}-k}$.

But if so, then the expression in (9) is the sum of a term that is less than 1 and $k$ nonpositive terms. Therefore expression in (9) must be weakly less than 1 and hence the claim that $A$ will benefit by replacing $B$ as a proposer in the last period of the cycle is true.

Now, if it is $A$ who is the proposer in the last period, we can always find the last period in which $B$ was a proposer (since $\pi$ is onto), swap $A$ and $B$ in these two periods, thus (by the result in subsection 4.3) making $A$ better off and then repeat the above argument. Hence, from these two observations we can conclude that if $A$ replaces $B$ as a proposer in the last period in which $B$ was the proposer, then $A$ will benefit from such a change.

But now, we can invoke the result from subsection 4.3 again and argue that since $A$ is better off by replacing $B$ as a proposer in the last period in which $B$ was a proposer, then also $A$ will be better off when replacing $B$ as a proposer in any period. The reason for this is the following: let $\pi$ be the original protocol, $\pi_{1}$ be the protocol formed from $\pi$ by replacing $B$ by $A$ in the last period when $B$ was a proposer and $\pi_{2}$ the protocol formed from $\pi_{1}$ by swapping proposers in the period in which we made change from $\pi$ to $\pi_{1}$ and the period in which we want the actual change to take place. Notice that by the reasoning we gave in this subsection, $\pi_{1}$ is better for $A$ than $\pi$ and by the result from subsection $4.3 \pi_{2}$ is better for $A$ than $\pi_{1}$ and hence $\pi_{2}$ is better for $A$ than $\pi$ and the 
only difference between these two protocols is that $A$ is a proposer one more time under $\pi_{2}$ than under $\pi$.

Obviously, by iterating this argument we can easily show that if we add more than one period to $P_{A}$, player $A$ will benefit from such a change. In particular, player $A$ will be best off if $\mathrm{P}=P_{A}$ (i.e. player $A$ is the proposer in every period of the game).

4.5. Normalization of the pie size does not matter. Finally, as already noted at the beginning, although the size of the pie is assumed to be equal to 1, this assumption is not crucial to the results we obtained. In principle, we could have any size $D$ of the pie that players bargain over as long as $D>0$ and rather than treat $x$ and $y$ as absolute sizes, we could treat them as the shares in the pie of any given size. That is, with the pie of size $D$ and under an offer $(x, y)$, player $A$ would get $x D$ and player $B$ would get $y D$ of the pie in absolute terms. Hence, it is clear that the assumption that the pie has the size 1 is just a matter of normalization and that if we vary the size of the pie, all equilibrium payoffs $z_{i}$ will just vary proportionately.

5. Conclusions and possible extensions. We have shown what form the equilibrium will take in the simplest case if we allow for general form of a protocol and different discount factors. There are a number of ways in which this result can be further extended:

- We can allow for non-zero outside options. Typically in the practical applications the negotiations need not last forever but may be terminated at some point in which case players get some (status quo) outside options. We can modify the model in such a way that following every rejection, the nature with some probability decides whether the game will continue or terminate, in which case both players would receive some known payoffs.

- As we know from bargaining experiments, the assumption that players are selfish and care only about their own payoffs is rather simplifying. In fact we observe players who decide to share the pie even in dictator games, when there is no threat of the offer being rejected. We can extend the model to allow for more general form of preferences, in which players' utilities would depend on both $x$ and $y$.

- Looking for the perfect Bayesian-Nash equlibrium of the game extended to one in which there is some uncertainty about discount factors of the players would also be an interesting extension, leading probably to the delay in the agreement being reached in an equilibrium. However, allowing for imperfect information in the game of infinite horizon in which proposers may choose from infinitely (and uncountably) many decision every time the split is offered, may turn out to complicate the game to a degree when it is not tractable analytically.

\section{References}

[1] S. Baliga and S. Serrano, Multilateral bargaining with imperfect information, Journal of Economic Theory 67 (1995), 578-589.

[2] K. Binmore and P. Dasgupta, The Economics of Bargaining, Basil Blackwell, Oxford, 1987. 
[3] S. Chae and J.-A. Yang, An N-person pure bargaining game, Journal of Economic Theory 62 (1994), 86-102.

[4] C.-Y. Huang, Multilateral bargaining: conditional and unconditional offers, Econom. Theory 20 (2002), 401-412.

[5] V. Krishna and R. Serrano, Multilateral bargaining, Review of Economic Studies 63 (1996), 61-80.

[6] A. Muthoo, Bargaining Theory with Applications, Cambridge University Press, 1999.

[7] A. Rubinstein, Perfect equilibrium in a bargaining model, Econometrica 50 (1982), 97-119.

[8] A. Shaked and J. Sutton, Involuntary unemployment as a perfect equilibrium in a bargaining model, Econometrica 52 (1984), 1351-1364.

[9] I. Ståhl, Bargaining Theory, Stockholm School of Economics, Stockholm, 1972.

[10] J. Sutton, Non-cooperative bargaining theory: An introduction, Review of Economic Studies 53 (1986), 709-724. 